\title{
Biologie du processus métastatique
}

> La formation de métastases est un événement critique de l'histoire naturelle d'un cancer et elle s'accompagne d'un pronostic clinique sombre. Mécanistiquement, le processus métastatique est un ensemble d'étapes distinctes, assez bien caractérisées et qui mettent en jeu de nombreux effecteurs moléculaires. Plusieurs modèles expliquant leur genèse ont été proposés : modèles sélectifs (sélection clonale) et adaptatifs (oncogenèse initiale), rôle de cellules souches tumorales, transition épithéliomésenchymateuse, etc. L'étude des cellules tumorales circulantes et disséminées, qui sont deux nouvelles fenêtres d'étude clinique et biologique du processus métastatique, devrait permettre de futures avancées. <

Historiquement, le terme métastase, du grec metastasis « je change de place », est apparu au XvI siècle pour désigner les tumeurs secondaires à une tumeur primitive. Ce phénomène est individualisé en tant qu'objet de recherche à partir de 1889 , date de la publication dans le Lancet par J. Paget de l'hypothèse « seed and soil » [1]. Le processus métastatique est actuellement défini comme « une dissémination de cellules néoplasiques dans un site secondaire (ou de plus grand ordre) non contigu et distant, au sein duquel ces cellules prolifèrent pour former une masse extravasculaire de cellules incomplètement différenciées » [2]. Nous aborderons dans cette revue exclusivement les métastases par voie hématogène issues de tumeurs non hématologiques.

\section{Aspects mécanistiques}

La distance qui sépare la tumeur primitive de ses métastases implique le franchissement de plusieurs étapes par les cellules tumorales. Le nombre et la nature de ces étapes sont variables selon les auteurs, avec des données contradictoires, principalement sur les étapes de circulation et d'extravasation. Nous rappelons brièvement, pour chacune de ces étapes, les principaux effecteurs moléculaires impliqués (Figure 1).

\section{François-Clément Bidard ${ }^{1}$, Marie-France Poupon ${ }^{2}$}

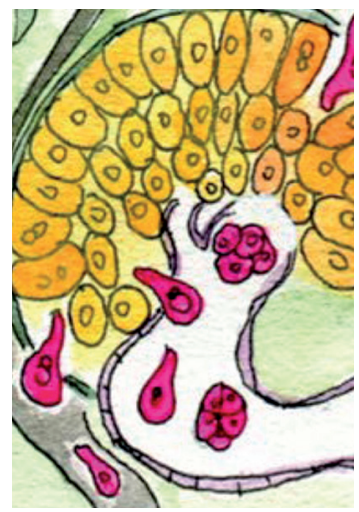

${ }^{1}$ Département d'oncologie médicale, Institut Curie, 26 rue d'Ulm, 75005 Paris ; université Paris Descartes, Paris ; ${ }^{2}$ Xentech, Génopole, 4 rue Pierre Fontaine, 91000 Evry, France fcbidard@curie.fr mariefrancepoupon@gmail.com

De multiples étapes à franchir pour les cellules tumorales métastatiques

\section{L'invasion locale : première étape du processus}

L'invasion locale fait intervenir principalement deux phénomènes intriqués : la dégradation du stroma péritumoral et la migration cellulaire. La dégradation de la matrice extracellulaire (MEC) est assurée par des protéases, notamment par le système de l'urokinase (uPA, urokinase-type plasminogen activator), de son récepteur (UPAR) et de ses inhibiteurs (PAI 1 et 2) [3]. Les métalloprotéases (MMP) et leurs inhibiteurs (TIMP) jouent aussi un rôle considérable dans la cascade protéolytique survenant au niveau du front d'invasion de la tumeur. Cette protéolyse permet une «activation » de la MEC, via la libération de nombreuses cytokines et facteurs de croissance (TGF $\beta$ [transforming growth factor], VEGF [vascular endothelial growth factor], etc.), qui ont alors un rôle trophique et chimioattractant pour les cellules tumorales. Des boucles de stimulations paracrines s'établissent de la même façon entre les cellules tumorales et les cellules stromales et immunitaires. Ce stroma activé comporte donc une activité protéolytique élevée, une angiogenèse accrue, une quantité importante de facteurs de croissance, un afflux de cellules inflammatoires et la présence de myofibroblastes. Tous ces facteurs concourent au développement tumoral initial [4]. Le processus de migration, second élément de l'invasion locale, correspond à une perte de l'adhésion intercellulaire qui s'accompagne de l'acquisition d'un phénotype migratoire : perte de la polarisation basolatérale au profit d'une polarisation de type front de migration/arrière. Cette perte de l'adhésion fait intervenir de très nombreuses molécules membranaires et/ou du cytosquelette. Par exemple, la perte de fonction de la $\varepsilon$-cadhérine (par protéolyse, mutation ou modification épigénétique) est corrélée au potentiel métastatique dans de nombreux cancers, notamment les 


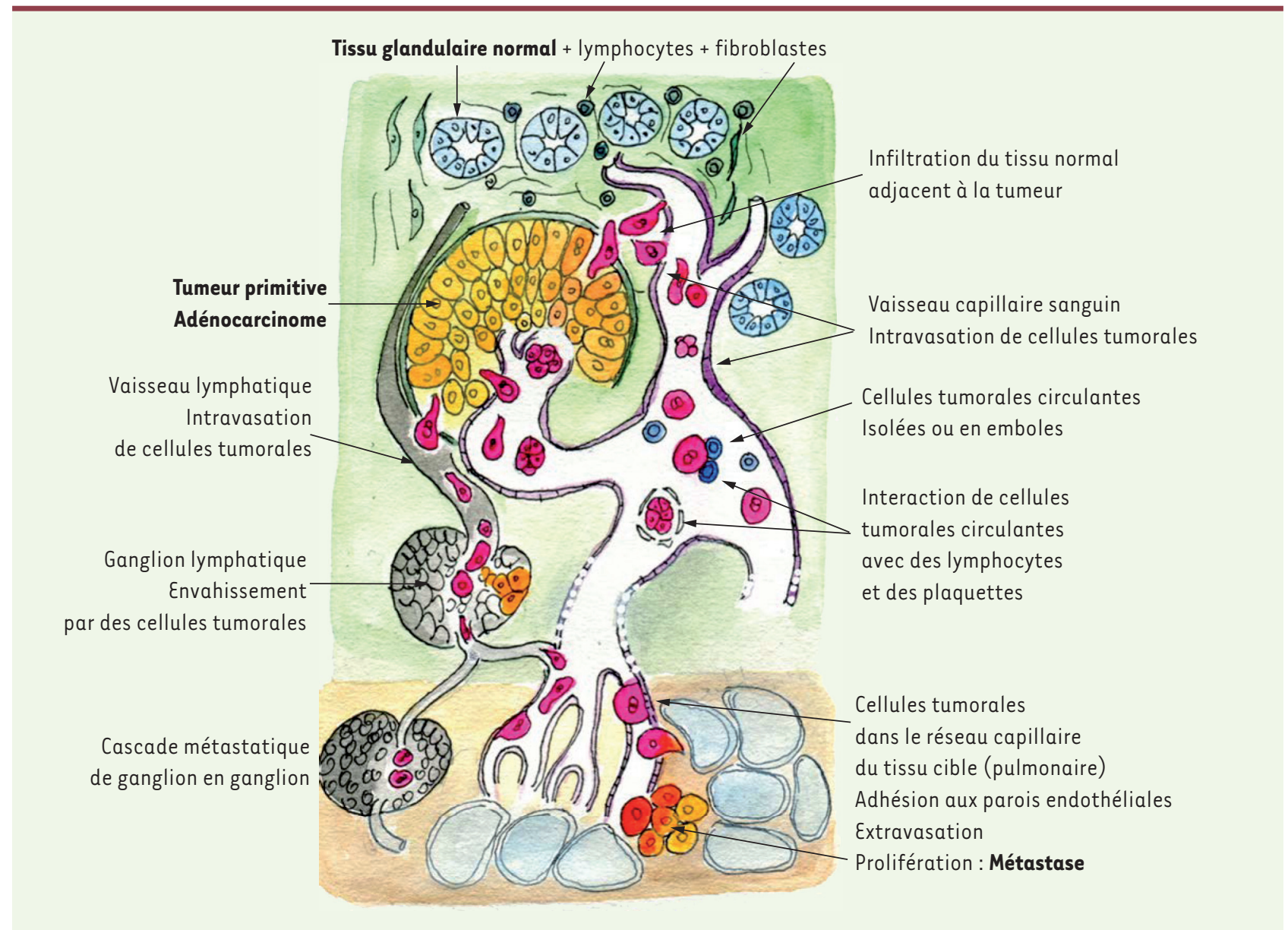

Figure 1. Les différentes étapes du processus métastatique. La métastase de cellules tumorales à partir de la tumeur primitive est possible, soit directement par voie hématogène, soit après invasion du tissu normal voisin, par voie lymphatique. La rupture de la membrane basale entourant les structures glandulaires peut être induite par les cellules tumorales. La voie lymphatique conduit les cellules tumorales de ganglions en ganglions vers la circulation sanguine, avec un risque d'implantation ganglionnaire, décrivant une partie de la cascade métastatique. Les cellules tumorales circulent également dans le sang, isolées ou en amas. Elles sont en contact avec les cellules du sang et avec les plaquettes, qui peuvent les agglutiner. Le ralentissement du flux sanguin dans les capillaires contribue à l'adhésion des cellules tumorales sur les parois endothéliales. L'intravasation des cellules tumorales et leur prolifération ont lieu dans le tissu cible métastatique, poumon, foie, cerveau, os, essentiellement. Un processus identique conduit les cellules tumorales à migrer de la métastase initiale vers d'autres sites (du tissu pulmonaire au cerveau, par exemple), complétant la cascade métastatique.

cancers colorectaux et mammaires. D'un point de vue moléculaire, la $\varepsilon$-cadhérine joue, via une interaction avec ses partenaires protéiques intracellulaires (p120, caténines), un rôle direct dans le remaniement de la morphologie cellulaire; via une signalisation intracellulaire multiple, elle est aussi responsable notamment de l'inhibition de contact. Autre exemple, les petites GTPases (Rho, Rac, etc.) jouent un rôle physiologique important dans l'organisation du cytosquelette, et plusieurs expériences d'analyse génomique comparative ont rapporté que la dérégulation de leur expression était associée au caractère métastatique de tumeurs primitives [5]. Il est important de noter qu'il existe plusieurs modes de migration cellulaire, qui peuvent être observés simultanément dans une même population cellulaire mais qui ne font pas appel aux mêmes mécanismes moléculaires : migration collective en feuillets (maintien des liaisons intercellulaires, similaire à la migration lors des mécanismes de réparation des plaies), et migration individuelle, qu'elle soit mésenchymateuse (cellule allongée à morphologie fibroblastique) et/ou amœboïde (cellule à morphologie ronde) [6].

\section{Circulation dans le sang}

L'entrée des cellules dans la lumière d'un vaisseau sanguin, ou intravasation, marque ensuite le début d'une étape brève et difficile à étudier : la circulation des cellules tumorales dans le sang et leur interaction avec les éléments figurés du sang. Cette circulation, 
qui peut se faire sous la forme de cellules isolées et/ou d'amas cellulaires (embols), nécessite des propriétés de résistance mécanique aux pressions élevées, ainsi qu'une résistance à l'apoptose induite par la perte d'un substrat d'adhésion (ce type d'apoptose est appelé anoïkis). L'arrêt des cellules tumorales circulantes se fait de manière soit passive dans un capillaire de petit calibre (effet «filtre » du foie et des poumons notamment), soit de manière active. Dans ce cas, il doit exister des mécanismes de reconnaissance spécifique et d'arrêt sur l'endothélium similaires à ceux qu'utilisent les leucocytes [38]. Cette dernière reconnaissance fait intervenir des protéines d'adhésion membranaires sur les cellules tumorales et endothéliales, ces protéines jouent un rôle probablement important dans l'existence de sites métastatiques préférentiels pour chaque type de cancer. Cette spécificité tissulaire des localisations métastatiques est un phénomène très connu en clinique et que décrivait Paget grâce à une comparaison botanique, les «graines » (seeds) de tumeur se développant dans un « terreau » favorable (soil) [1]. L'existence de niches prémétastatiques au niveau des organes cibles, au sein desquelles les cellules tumorales circulantes s'arrêteraient préférentiellement a été rapportée dans un modèle expérimental [7], mais peu de preuves cliniques sont venues conforter ces résultats jusqu'à présent [8].

\section{L'extravasation et la dormance des cellules tumorales}

La migration à travers l'endothélium, ou extravasation, est alors l'étape suivante observée dans les expériences de vidéomicroscopie (quoiqu'inconstante [9]). Elle est suivie d'une nouvelle interaction avec le microenvironnement de l'organe hôte, qui peut être très différent de celui du site primitif. II peut survenir alors une étape de dormance cellulaire, pendant laquelle les cellules tumorales disséminées persistent sans proliférer pendant un temps indéterminé, avant de reprendre - ou non - une nouvelle croissance aboutissant à l'apparition d'une métastase. Les mécanismes qui régulent l'entrée et la sortie de la phase de dormance restent obscurs, du fait de la difficulté de modéliser cette étape dans des modèles précliniques murins. S'agit-il d'une interaction avec le système immunitaire [39] ? D'une réversion du switch angiogénique? D’un effet inhibiteur du nouveau microenvironnement tumoral [10]?

Plus récemment, un niveau supérieur de complexité a été rapporté, avec la mise en évidence d'une recirculation des cellules tumorales des sites distants vers le sang et la tumeur primitive. Cet autoensemencement (self seeding) favoriserait la croissance de l'ensemble des sites tumoraux, y compris celui de la tumeur primitive [11].

Les étapes citées ci-dessus font intervenir un nombre important d'effecteurs moléculaires différents, et, pour une cellule tumorale donnée, la probabilité de pouvoir franchir avec succès toutes ces étapes est extrêmement faible. D'anciennes expériences ont montré que le taux de formation de métastases à partir de cellules tumorales injectées dans la circulation serait de l'ordre de 1/1 000000 [12], et suggéraient donc une inefficacité métastatique des cellules tumorales. Les expériences de vidéomicroscopie in vivo rapportées au début des années $2000[9,13]$ indiquent que le processus métastatique semble être surtout sélectif au cours des dernières étapes (intravasation, régulation de la survie et de la dormance des cellules tumorales disséminées), mais ces données restent à confirmer en clinique.

\section{Déterminants moléculaires}

Les étapes du processus métastatique font appel à des effecteurs moléculaires multiples, très redondants, et qui sont souvent impliqués dans les propriétés développées préalablement par la tumeur primitive (angiogenèse, échappement au système immunitaire et à l'apoptose, invasion, etc.). Il est donc peu surprenant que les gènes dont les variations d'expression ont été associées à une variation du potentiel métastatique soient innombrables et n'aient eu pour la plupart aucune application clinique jusqu'à présent. J. Massagué [14] a proposé une classification en quatre catégories de cette multitude de gènes potentiellement impliqués dans le processus métastatique : les gènes tumorigéniques (oncogenèse et croissance initiale), les gènes des premières étapes de la métastase (motilité, invasion, angiogenèse et intravasation), les gènes de la progression métastatique (survie dans le sang, adhésion capillaire ciblée, extravasation, survie dans le microenvironnement secondaire) et les gènes de la virulence métastatique (reprise de la croissance tumorale secondaire). En clinique, les gènes les plus fréquemment associés au risque de métastase sont en grande majorité des gènes tumorigéniques, liés à la prolifération de la tumeur primitive [15], et non pas ceux qui codent pour les effecteurs d'une étape particulière du processus métastatique.

\section{Transition épithéliomésenchymateuse (عMT)}

L'EMT est définie comme le processus au cours duquel une cellule épithéliale : (1) acquiert un phénotype invasif, fibroblastoïde ; (2) diminue l'expression de protéines spécifiquement épithéliales et exprime des protéines spécifiquement mésenchymateuses (vimentine, $\mathrm{N}$-cadhérine, etc.) ; (3) dégrade et migre à travers la MEC. L'EMT est un processus cellulaire intervenant dans plusieurs phases du développement embryonnaire normal, notamment au stade de blastula par la formation du mésoderme lors de la gastrulation [16]. Compte tenu des propriétés migratoires des cellules mésenchymateuses, l'EMT pourrait être un moyen, pour les cellules tumorales épithéliales, d'acquérir un potentiel métastatique. II a été ainsi rapporté dans de nombreux modèles que la progression tumorale vers un stade invasif et métastatique s'associe à une dédifférenciation cellulaire, avec altération de la polarisation et acquisition d'un morphotype fibroblastoïde [17]. Au niveau moléculaire, la détection des marqueurs 
signant I'EMT est moins probante ; il suffit par exemple de rappeler que la détection des cytokératines épithéliales dans les cellules de métastases est utilisée quotidiennement pour orienter la recherche de l'origine du cancer primitif. Quelques travaux décrivent cependant une coexpression de marqueurs épithéliaux et mésenchymateux dans les cellules métastatiques [18]; d'autres proposent que l'EMT ne soit qu'un processus transitoire, essentiellement présent au niveau du front d'invasion de la tumeur primitive, et que les cellules micrométastatiques se redifférencient en cellules épithéliales (transition mésenchymoépithéliale) une fois installées dans le site métastatique, sous l'effet d'un microenvironnement différent [19]. Cette acquisition transitoire du phénotype mésenchymateux pourrait en outre conférer aux cellules des propriétés de cellules souches tumorales, capables de reformer à distance une nouvelle tumeur [20]. Mécanistiquement, plusieurs voies de signalisation ont été décrites comme responsables de l'acquisition d'un potentiel métastatique via l'EMT: TGF-bêta, NF-kappaB, Twist [21, 40] ; ces voies peuvent être stimulées lors des phases d'invasion locale.

\section{Déterminants génétiques}

\section{Théorie sélective}

L'expérience princeps de la théorie de la sélection clonale rapportée par Fidler en 1977 [22] montrait que l'on pouvait enrichir, par sélections successives, une population cellulaire initialement faiblement métastatique en cellules fortement métastatiques. Les métastases seraient donc issues d'une sous-population cellulaire au sein de la tumeur initiale, responsable à elle seule de la croissance métastatique. Dans l'autre expérience fondatrice [23], inspirée du test de fluctuation de Luria et Delbrück utilisé en bactériologie, le potentiel métastatique de sous-clones dérivés d'une population cellulaire initiale a été testé. Ce potentiel s'est révélé très hétérogène (comparaison statistiquement significative), suggérant que la capacité de métastaser caractérise une sous-population préexistante au sein de la tumeur primitive, et ne reflète pas une adaptation des cellules tumorales primitives.

La théorie de la sélection clonale fait donc reposer l'apparition de métastases sur la présence, au sein de la population tumorale primitive, d'au moins une sous-population possédant tous les déterminants génétiques et phénotypiques nécessaires au processus métastatique. Si le moment de l'acquisition du potentiel métastatique n'est pas déterminé [24], cela conduit implicitement à rechercher le $(s)$ gène $(s)$ qui serai(en)t responsable(s) d'un switch métastatique, à l'instar du switch angiogénique. Ce(s) gène(s) verrai(en)t son(leur) expression dérégulée, après l'oncogenèse initiale, par l'instabilité génétique connue des cellules tumorales. Ce switch métastatique serait ainsi un évènement rare dont la probabilité de survenue dans une tumeur serait liée au nombre de cellules tumorales, et donc à la taille de la tumeur. Dans ce modèle de switch, plusieurs gènes peuvent être impliqués, parmi lesquels on peut distinguer des gènes maîtres régulateurs et des gènes effecteurs. Les gènes régulateurs seraient des facteurs de transcription ou de signalisation, et seraient les initiateurs de la dérégulation de l'expression des gènes secondaires, effecteurs. Ces derniers coderaient pour l'ensemble des protéases et molécules d'adhésion décrites précédemment et nécessaires au franchissement successif des étapes du processus métastatique.

Cette théorie a donc été à l'origine, au cours des années 1980 à 1990, d'un effort important de recherche «du » gène responsable du switch métastatique. Comme nous l'avons déjà mentionné, la plupart des gènes « inducteurs » ou « répresseurs » de métastases qui ont été alors décrits agissent en réalité sur la croissance de la tumeur primitive (prolifération, invasion, etc.). Néanmoins, une dizaine de gènes appelés «suppresseurs de métastases »a été isolée, car inhibant la capacité métastatique de la tumeur sans agir sur sa vitesse de croissance initiale : Nm23 [41], Brmsl, Kissl, entre autres. Ces gènes ont fait l'objet d'études plus approfondies depuis les années 1990, mais leur caractère pronostique chez les patients reste, 20 ans plus tard, toujours sujet à débat [25].

La théorie de la sélection clonale semble être actuellement confortée par les premières données des techniques de séquençage à haut débit qui suggèrent une origine clonale des métastases, même si le caractère minoritaire $[26,27]$ ou non [28] au sein de la tumeur primitive du sous-clone métastatique reste discuté. En clinique, cette théorie suggère qu'il peut exister des différences marquées entre tumeur primitive et métastases, concernant notamment la présence des cibles moléculaires des nouvelles thérapeutiques (par exemple les inhibiteurs de mTOR [mamalian target of rapamycin]). Ces discordances semblent concerner avant tout les anomalies moléculaires acquises secondairement à l'oncogenèse, alors que les anomalies initiales ayant participé à l'oncogenèse (par exemple k-ras dans le cancer colorectal) ne semblent que peu concernées, du fait de l'apparente dépendance (addiction) des cellules tumorales à ces événements oncogéniques initiaux.

\section{Les théories adaptatives}

Le modèle d'évolution parallèle a été proposé par C.A. Klein au début des années 2000. Ce modèle se fonde sur l'observation que les cellules tumorales disséminent de manière très précoce au cours de l'histoire naturelle de la tumeur (et non pas de manière tardive après acquisition du switch métastatique), et que les modifications génétiques portées par ces cellules tumorales disséminées (ou micrométastases) sont limitées et varient d'une cellule à l'autre, et ne sont donc pas clonales [24]. Cette théorie propose 
finalement d'attribuer la latence d'apparition des métastases à la lenteur de l'évolution génétique des cellules tumorales disséminées dont l'activité réplicative est nettement plus modérée (dormance) que celle des cellules de la tumeur dont elles sont issues. Ce modèle reste néanmoins hypothétique, car il se fonde sur des analyses génomiques sur cellule unique, qui peuvent être à l'origine de possibles artéfacts.

Le modèle d'oncogenèse initiale est proposé par $\mathrm{R}$. Weinberg depuis le milieu des années 2000 et constitue actuellement l'alternative la plus reconnue au modèle sélectif. Dans ce modèle, la capacité métastatique d'une tumeur donnée est liée à la propension des cellules tumorales à réactiver le programme transcriptionnel de l'EMT [29]. La théorie proposée est que la sélection darwinienne s'exerce sur l'ensemble des cellules tumorales jusqu'au stade tumoral préinvasif. Après l'invasion de la membrane basale, l'interaction avec le stroma péritumoral activé (voir ci-dessus) est responsable de l'induction d'une EMT dans les cellules de la périphérie tumorale, via différentes cytokines [30]. Si l'on considère que l'acquisition d'un phénotype mésenchymateux rend les cellules tumorales métastatiques, la capacité à former des métastases serait finalement un processus induit (par l'évolution et l'invasion tumorale locale) et donc adaptatif. L'un des principaux arguments qui sous-tend cette théorie est le fait que l'analyse transcriptomique des tumeurs primitives permet, dans de très nombreux types tumoraux, de prédire l'évolution métastatique. Il a aussi été démontré en clinique que ces signatures moléculaires étaient conservées entre tumeur primitive et métastases [31]. Ce type d'observation ne correspond pas au modèle

Prélèvement : prise de sang (CTC) ou aspiration de moelle osseuse (CTD)

Délai maximal avant analyse très variable selon les techniques:
quelques minutes/heures à quelques jours

Enrichissement pré-analytique (selon les techniques)

Immunoenrichissement en cellules épithéliales

Séparation par taille

Séparation par déformabilité

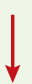

Marquage (selon les techniques)

Coloration non spécifique (HE, DAPI, etc.)

Immunomarquage épithélial (cytokératines, etc.)

Contre-immunomarquage des leucocytes (CD45, etc.)

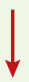

Détection (selon les techniques)

Détection automatisée ou manuelle

* Contrôle de la morphologie tumorale

Figure 2. Méthodes de détection des cellules tumorales circulantes et des cellules tumorales disséminées. sélectif selon lequel les caractéristiques transcriptomiques du sous-clone tumoral métastatique sont diluées au sein de la tumeur primitive par celles des autres sous-clones tumoraux.

Plusieurs autres modèles d'inspiration adaptative, coopération intercellulaire [32] et dominance clonale [33], ont été proposés mais restent insuffisamment étayés à ce jour. Plusieurs expériences précliniques suggèrent par ailleurs que les métastases pourraient être issues de cellules souches tumorales [34], mais il n'existe que peu d'arguments cliniques jusqu'à présent. Le rôle des polymorphismes génétiques liés à l'hôte reste aussi incomplètement documenté.

\section{Deux nouveaux champs d'étude clinique : les cellules tumorales circulantes et les cellules tumorales disséminées}

Chez l'homme, la biologie du processus métastatique a longtemps reposé sur l'analyse de ses deux extrémités : tumeurs primitives et métastases macroscopiques. Les techniques très sensibles de biologie moléculaire (RT-PCR) et, plus récemment, d'immunocytomarquage, généralement précédées d'un immunoenrichissement des cellules tumorales (Figure 2), ont permis l'ouverture de deux fenêtres supplémentaires d'étude : le sang périphérique et les organes hôtes potentiels. Ces deux sites de détection correspondent à des niveaux différents d'avancée le long du processus métastatique, et pourraient avoir des valeurs pronostiques différentes. Les cellules tumorales circulantes (CTC), détectées dans le sang périphérique, ont a priori une signification biologique assez simple : il s'agit de cellules tumorales ayant passé l'étape d'intravasation, et qui sont en transit dans le sang, avec une demi-vie relativement courte. Les cellules tumorales disséminées (CTD, ou micrométastases), qui correspondent à l'étape post-extravasation, peuvent être recherchées dans tous les tissus potentiellement hôtes d'une future croissance métastatique. La valeur pronostique des CTC et des CTD s'avère dorénavant importante dans la plupart des types tumoraux étudiés, tant en situation 
métastatique qu'en situation adjuvante $[35,36]$. La caractérisation moléculaire de ces cellules tumorales est actuellement un enjeu majeur pour la compréhension des étapes post-extravasation; cette même caractérisation moléculaire des CTC est actuellement à l'étude en clinique pour déterminer si ces cellules présentent les mêmes caractéristiques moléculaires que les masses tumorales ou s'il existe des différences (lesquelles pourraient être l'objet de traitements spécifiques, par exemple l'activation significative d'une cible moléculaire lors du passage dans la circulation). Biologiquement, l'appréciation de ces différences, ainsi que la mesure de l'hétérogénéité des cellules circulantes/disséminées pourraient permettre de fournir des arguments supplémentaires en faveur des théories sélectives ou adaptatives citées précédemment.

\section{Conclusion}

La mécanique du processus métastatique a été particulièrement étudiée au cours de ces trente dernières années et les progrès récents ont permis de remettre en cause, au moins partiellement, la théorie de la sélection clonale au profit d'une théorie adaptative d'induction de l'EMT par le microenvironnement. Cette accumulation de données précliniques, notamment sur les gènes régulateurs du processus métastatique, a pour objectif - encore non atteint - de permettre le développement de traitements inhibiteurs du processus métastatique. Les premiers essais réalisés dans ce domaine avec les inhibiteurs de métalloprotéases [37] ont clairement montré que ces médicaments potentiels doivent faire l'objet de programmes de développement clinique spécifiques, différents de ceux des anticancéreux classiques, qui tiennent compte de la longueur de ce processus (souvent plusieurs années) et des difficultés propres à la recherche clinique en situation adjuvante. L'étude directe des CTC et des CTD chez les patients est l'une des pistes actuelles qui pourrait permettre, au-delà de l'accroissement des connaissances, de répondre à des questions cliniques pour lesquelles les modèles expérimentaux ne sont pas adaptés à ce jour. $\diamond$

\section{SUMMARY}

The metastatic process: history, models and recent advances

The onset of metastasis is a critical event in the natural history of cancer, and is generally associated with a poor clinical outcome. Mechanistically, the metastatic process is made of several steps that are biologically distinct and now rather well characterized. Several explanatory models have been proposed: selective models (clonal selection), adaptive models (initial oncogenesis), involvement of tumor "stem" cells, epithelial-mesenchymal transition... The next progresses are expected to come from the characterization of circulating and disseminated tumor cells, which are two recently opened windows on the metastatic process in patients. $\diamond$

\section{CONFLIT D'INTÉRÊTS}

Les auteurs déclarent n'avoir aucun conflit d'intérêts concernant les données publiées dans cet article.

\section{RÉFÉRENCES}

1. Paget $\mathrm{S}$. The distribution of secondary growths in cancer of the breast. 1889. [1 $1^{\text {re }}$ publication. Lancet $1889 ; 133: 571-3$.] Cancer Metastasis Rev $1989 ; 8$ : 98-101.

2. Welch DR. Do we need to redefine a cancer metastasis and staging definitions? Breast Dis 2006-2007; $26: 3-12$.

3. Egeblad M, Werb Z. New functions for the matrix metalloproteinases in cancer progression. Nat Rev Cancer 2002 ; 2 : 161-74.

4. Finak G, Laferrière J, Hallett M, Park M. Le microenvironnement tumoral : un nouveau paramètre pronostique dans le cancer du sein. Med Sci (Paris) $2009 ; 25: 439-41$.

5. Clark $\varepsilon A$, Golub TR, Lander $\varepsilon S$, Hynes RO. Genomic analysis of metastasis reveals an essential role for RhoC. Nature $2000 ; 406: 532-5$.

6. Friedl P, Wolf K. Tumour-cell invasion and migration: diversity and escape mechanisms. Nat Rev Cancer $2003 ; 3: 362-74$.

7. Kaplan RN, Psaila B, Lyden D. Bone marrow cells in the "pre-metastatic niche": within bone and beyond. Cancer Metastasis Rev 2006 ; 25 : 521-9.

8. Mimori K, Fukagawa T, Kosaka Y, et al. Hematogenous metastasis in gastric cancer requires isolated tumor cells and expression of vascular endothelial growth factor receptor-1. Clin Cancer Res 2008 ; 14 : 2609-16.

9. Al-Mehdi AB, Tozawa K, Fisher AB, et al. Intravascular origin of metastasis from the proliferation of endothelium-attached tumor cells: a new model for metastasis. Nat Med $2000 ; 6: 100-2$.

10. Aguirre-Ghiso JA. Models, mechanisms and clinical evidence for cancer dormancy. Nat Rev Cancer $2007 ; 7: 834-46$.

11. Kim My, Oskarsson T, Acharyya S, et al. Tumor self-seeding by circulating cancer cells. Cell 2009 ; 139 : 1315-26.

12. Fidler IJ. Metastasis: quantitative analysis of distribution and fate of tumor embolilabeled with 125 I-5-iodo-2'-deoxyuridine. J Natl Cancer Inst 1970 ; $45: 773-82$.

13. Naumov GN, MacDonald IC, Weinmeister PM, et al. Persistence of solitary mammary carcinoma cells in a secondary site: a possible contributor to dormancy. Cancer Res $2002 ; 62: 2162-8$.

14. Nguyen DX, Bos PD, Massagué J. Metastasis: from dissemination to organspecific colonization. Nat Rev Cancer $2009 ; 9: 274-84$.

15. Andre F, Delaloge $S$. First-generation genomic tests for breast cancer treatment. Lancet Oncol $2010 ; 11: 6-7$.

16. Thiery JP, Sleeman JP. Complex networks orchestrate epithelial-mesenchymal transitions. Nat Rev Mol Cell Biol 2006 ; 7 : 131-42.

17. Thiery JP. Epithelial-mesenchymal transitions in tumour progression. Nat Rev Cancer $2002 ; 2: 442-54$.

18. Oft M, Akhurst RJ, Balmain A. Metastasis is driven by sequential elevation of H-ras and Smad2 levels. Nat Cell Biol $2002 ; 4: 487-94$.

19. Brabletz $T$, Jung $A$, Reu $S$, et al. Variable beta-catenin expression in colorectal cancers indicates tumor progression driven by the tumor environment. Proc Natl Acad Sci USA 2001 ; 98 : 10356-61.

20. Mani SA, Guo W, Liao MJ, et al. The epithelial-mesenchymal transition generates cells with properties of stem cells. Cell $2008 ; 133: 704-15$.

21. Yang J, Mani SA, Donaher JL, et al. Twist, a master regulator of morphogenesis, plays an essential role in tumor metastasis. Cell $2004 ; 117$ : 927-39.

22. Fidler IJ, Kripke ML. Metastasis results from preexisting variant cells within a malignant tumor. Science $1977 ; 197: 893-5$

23. Hill RP, Chambers AF, Ling V, Harris JF. Dynamic heterogeneity: rapid generation of metastatic variants in mouse Bl6 melanoma cells. Science $1984 ; 224$ : 998-1001.

24. Schmidt-Kittler 0 , Ragg T, Daskalakis A, et al. From latent disseminated cells to overt metastasis: genetic analysis of systemic breast cancer progression. Proc Natl Acad Sci USA 2003 ; 100 : 7737-42.

25. Steeg PS. Metastasis suppressors alter the signal transduction of cancer cells. Nat Rev Cancer ; $3: 55-63$.

26. Ding L, Ellis MJ, Li S, et al. Genome remodelling in a basal-like breast cancer metastasis and xenograft. Nature $2010 ; 464: 999-1005$.

27. Yachida $S$, Jones $S$, Bozic I, et al. Distant metastasis occurs late during the genetic evolution of pancreatic cancer. Nature $2010 ; 467$ : 1114-7.

28. Navin N, Kendall J, Troge J, et al. Tumour evolution inferred by single-cell sequencing. Nature $2011 ; 472: 90-4$.

29. Hartwell KA, Muir B, Reinhardt F, et al. The Spemann organizer gene, Goosecoid, promotes tumor metastasis. Proc Natl Acad Sci USA 2006 ; 103 : 18969-74.

30. Karnoub AE, Dash AB, Vo AP, et al. Mesenchymal stem cells within tumour stroma promote breast cancer metastasis. Nature $2007 ; 449$ : 557-63. 


\section{RÉFÉRENCES}

31. Weigelt B, Hu Z, He X, et al. Molecular portraits and 70-gene prognosis signature are preserved throughout the metastatic process of breast cancer. Cancer Res $2005 ; 65: 9155-8$.

32. Bidard FC, Pierga JY, Vincent-Salomon A, Poupon MF. A "class action" against the microenvironment: do cancer cells cooperate in metastasis? Cancer Metastasis Rev 2008 ; 27 : $5-10$.

33. Kerbel RS, Waghorne C, Korczak B, et al. Clonal dominance of primary tumours by metastatic cells: genetic analysis and biological implications. Cancer Surv $1988 ; 7: 597-629$

34. Häfner S, Coulombel L. L'oligarchie contestée des cellules souches cancéreuses. Med Sci (Paris) $2009 ; 25: 227-8$.

35. Bidard FC, Ferrand FR, Huguet F, et al. Disseminated and circulating tumor cells in gastrointestinal oncology. Crit Rev Oncol Hematol 2011 ; doi:10.1016/j.critrevonc.2011.05.008

36. Mego M, Mani SA, Cristofanilli M. Molecular mechanisms of metastasis in breast cancer - clinical applications. Nat Rev Clin Oncol 2010; $7: 693-701$.
37. Coussens LM, Fingleton B, Matrisian LM. Matrix metalloproteinase inhibitors and cancer: trials and tribulations. Science $2002 ; 295: 2387-92$.

38. Le Cabec V, Guiet R, Maridonneau-parini I, van goethem $\varepsilon$. La migration des phagocytes : tour d'horizon. Med Sci (Paris) $2011 ; 27$ : 1112-9.

39. Quesnel B. Je te tiens, tu me tiens... Dormance tumorale : un équilibre instable? Med Sci (Paris) $2008 ; 24: 575-6$.

40. Puisieux A, Ansieau S. La dissémination précoce de cellules métastatiques : une nouvelle donnée aux conséquences majeures? Med Sci (Paris) 2009 $25: 28-9$

41. Boissan M, Poupon MF, Lacombe ML. NM23 et les gènes suppresseurs de métastases. Med Sci (Paris) $2007 ; 23: 1115-23$

\section{TIRÉS À PART}

M.F. Poupon

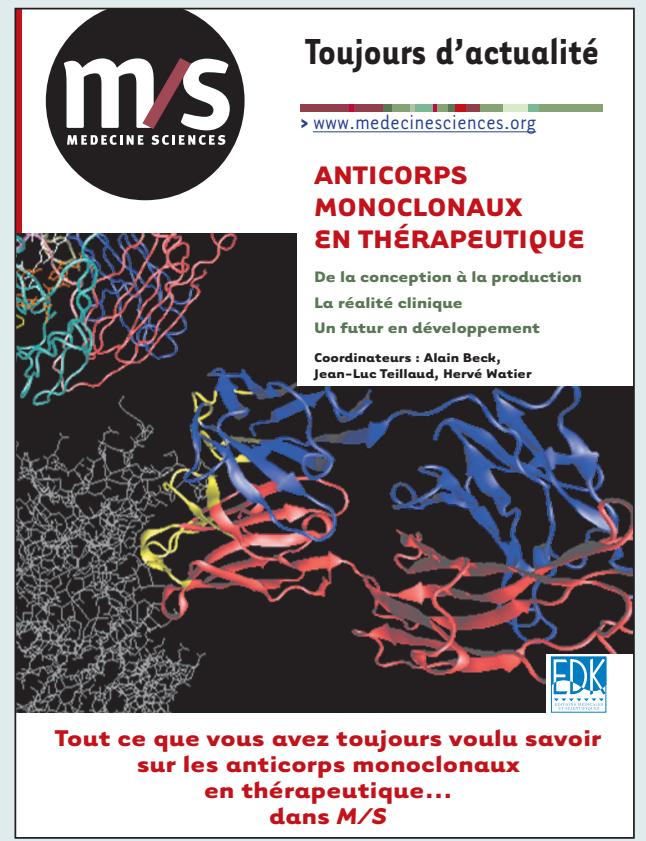

Tout ce que vous avez toujours voulu savoir sur les anticorps monoclonaux en thérapeutique... dans Médecine/Sciences. Pourquoi un numéro spécial de Médecine/Sciences sur les anticorps monoclonaux thérapeutiques? II nous a semblé que le moment était venu de dresser un état des lieux de ces biomédicaments qui prennent désormais une place considérable - et croissante dans les traitements de maladies souvent lourdes et désespérantes. Ce voyage que nous vous proposons à la découverte du monde des anticorps thérapeutiques nous a appris, ou plutôt rappelé, une évidence : les compétences en France sont fortes et nombreuses, qu'elles soient académiques ou industrielles, biotechnologiques ou cliniques. Le paysage français, trop longtemps discret, bruisse désormais de mille initiatives balayant de multiples aspects des anticorps thérapeutiques : études précliniques et cliniques menées avec de nouveaux anticorps dirigés contre des cibles originales, développement de nouveaux formats d'anticorps ou d'anticorps optimisés reposant sur des études structurales et fonctionnelles sophistiquées, recherche active de cibles pertinentes, mise au point de méthodologies de bioproduction, de couplage, etc. L'expansion industrielle rapide de ce champ est un défi que peut et doit relever notre pays, défi tant scientifique qu'économique, avec ses combats pour la propriété intellectuelle et pour l'emploi de nos jeunes scientifiques.

Alain Beck, Jean-Luc Teillaud, Hervé Watier

\section{) À retourner à EDK, 25, rue Daviel - 75013 Paris, France}

Tél. : 0158101905 - Fax : 0143293262 - E-mail : edk@edk.fr

NOM :

Prénom :

Adresse :

Code postal :

Ville :

Pays :

Fonction :

Je souhaite recevoir M/S nº 12 - décembre 2009 (Anticorps monoclonaux en thérapeutique) : $25 €+3 €$ de port $=\mathbf{2 8} €$ TTC en ................. exemplaire, soit un total de $€$

Par chèque, à l'ordre de $\mathbf{E} \mathbf{D} \mathbf{K}$

Par carte bancaire : $\square$ Visa Eurocard/Mastercard Carte $n^{\circ}$

Date d'expiration :

$\mathrm{N}^{\circ}$ de contrôle au dos de la carte :

Signature : 